\title{
Combining Fractals and Box-Counting Dimension
}

\author{
M. Ndiaye \\ Mathematics and Computer Sciences Department, Marist College, Poughkeepsie, NY, USA \\ Email: malick.ndiaye@marist.edu
}

How to cite this paper: Ndiaye, M. (2021) Combining Fractals and Box-Counting Dimension. Applied Mathematics, 12, 818-834. https://doi.org/10.4236/am.2021.129055

Received: July 1, 2021

Accepted: September 27, 2021

Published: September 30, 2021

Copyright () 2021 by author(s) and Scientific Research Publishing Inc. This work is licensed under the Creative Commons Attribution International License (CC BY 4.0).

http://creativecommons.org/licenses/by/4.0/

\begin{abstract}
In this paper, the box-counting dimension is used to derive an explicit formula for the dimension of a fractal constructed using several contractions or by combining fractals. This dimension agrees with the Hausdorff dimension in the particular case when the scales factors considered are all the same. A more general sufficient condition for the box-counting dimension and the Hausdorff dimension to be the same is given. It is also shown that the dimension of the fractal obtained by combining two fractals is the weighted average of the dimensions of the two fractals.
\end{abstract}

\section{Keywords}

Box-Counting Dimension, Combining Fractals

\section{Introduction}

We can think of fractals as a complex geometric figure that is self similar across different scales. Fractals occupy the space between two discrete dimensions. These are shapes whose dimension is not a whole number unlike any Euclidean space. Since fractals show similar details on different scales, so scaling is at the center of understanding fractals dimension. When one successfully magnifies a section of a fractal image, each magnification reveals repeated fractals shapes that are similar in detail to the images of the main fractal and other magnification. There are various ways to construct fractals mathematically. We have for example a deterministic process like the one used to construct the classic Cantor set [1] [2] by iteratively deleting the middle third section of the unit interval. We can also construct fractals by repeatedly iterating a given map or through the use of IFS (Iterating Function System) [1] [3] or by looking at the basin boundary of infinity of the Mendelbrot set: Julia set [1] [4] [5] [6]. In their article: On combining and 
convolving fractals [7], the authors introduced a new process of constructing fractals: The convolution of fractals into one. They then explore the effect of such combinations on the dimensions of the corresponding fractals by using the Moran equations [8] [9]. In general, finding an explicit formula for the dimension in term of elementary functions is quite difficult, and sometime impossible. In this paper, the box-counting dimension [2] [10] is used to derive an alternative explicit formula for the dimension of fractals constructed using several contractions or by combining fractals. The formula for the dimension of fractals constructed using several contractions could be seen as the generalization of the Cantor set dimension in the case we apply a multitude of scales on the unit interval. With this formula, it is much easier to show several properties of the dimension of the fractal obtained by combining two fractals than using the dimension from the Moran equation.

The paper is divided into six sections.

In Section 2, the definition of contractions, iterated functions system (IFS) are introduced. The existence of a limiting set from an IFS is discussed. An example of fractal is also given.

In Section 3, the definition and the properties of the box-counting dimension are introduced and specific examples about the box-counting dimension of the Cantor set and the Sierpinski gasket are given.

In Section 4, the box-counting dimension is applied to fractals that arise from multitude of scaling.

In Section 5, the dimension of fractals obtained by combination of two fractals is explored and it is shown that this dimension is a weighted average.

Section 6 is dedicated to the conclusion.

\section{Notion of Contractions, Iterated Function Systems, Fractal Definition}

The functions that are used in mathematics to reproduce a contracted self-similar image from a given figure is called contraction. This notion is defined as follow.

Definition 1. A contraction in $\mathbb{R}^{m}$ with scale factor $r \in(0,1)$ is a map $f: \mathbb{R}^{m} \rightarrow \mathbb{R}^{m}$ such that for all $x$ and $y$ in $\mathbb{R}^{m}|f(x)-f(y)| \leq r|x-y|$.

Remark 1. If $|f(x)-f(y)|=r|x-y|$ then $f$ is said to be a similarity with scale factor $r$.

Matrices provide a very convenient notation for contractions. A contraction can be written as $f(x)=A x+b$ where $x \in \mathbb{R}^{m}$ and $A$ is a $m \times m$ matrix whose eigenvalues have magnitudes less than 1 and $b$ is a constant in $\mathbb{R}^{m}$.

Definition 2. An iterated function system (IFS) is a collection of contractions $\left\{f_{1}, f_{2}, \cdots, f_{n}\right\}$ with $n \geq 2$ on a closed subset $\Omega \subset \mathbb{R}^{n}$. A nonempty compact subset $F$ of $\Omega$ is an attractor or fractal of the IFS if $F=\bigcup_{i=1}^{n} f_{i}(F)$.

The following theorem shows the existence and the uniqueness of such attractor given any IFS.

Theorem 1. Given any IFS defined by the contractions $C_{1}, C_{2}, C_{3}, \cdots, C_{m}$ on a 
closet subset $\Omega$ of $\mathbb{R}^{m}$ and let $\mathcal{C}$ be the set of all nonempty compact subsets of $\Omega$ and let $f$ be the map defined as $f(X)=\bigcup_{i=1}^{m} C_{i}(X)$ where $\mathcal{C}$, then there exists a unique attractor $F$ such that $f(F)=F$. Furthermore, for every set $X \in \mathcal{C}$ such that $f(X) \subset X, F=\bigcap_{i=1}^{\infty} f^{i}(X)$.

Proof. let $\Omega$ be a closed set in $\mathbb{R}^{m}$. Let $E \subset \Omega$.

The $\delta$-neighborhood of $E$ denoted $N_{\delta}(E)$ is defined as

$$
N_{\delta}(E)=\{x \in \Omega /|x-a|<\delta \text { for some } a \in E\} .
$$

Let $\mathcal{C}$ be the set of compact in $\Omega$.

The Hausdorff metric on $\Omega$ is defined as:

For any set $A, B$ in $\mathcal{C}: d_{H}(A, B)=\inf \left\{\delta / A \subset N_{\delta}(B), B \subset N_{\delta}(A)\right\}$.

- The set of compact $\mathcal{C}$ in $\Omega$ is a complete space using the Hausdorff metric.

Let $S_{n}$ be a Cauchy sequence in $\mathcal{C}$ that is $\forall \varepsilon>0 \quad \exists N \in \mathbb{N}$ such that if $n, m \geq N$ then $d_{H}\left(S_{n}, S_{m}\right)<\varepsilon$. $i \geq 1$

Choose $\varepsilon_{i}=\frac{\varepsilon}{2^{i}} \quad \exists N_{i} \in \mathbb{N}$ such that if $n, m \geq N$ then $d_{H}\left(S_{n}, S_{m}\right)<\frac{\varepsilon_{i}}{2^{i}}$,

If $N_{1}<N_{2}<N_{3}<\cdots<N_{i}<\cdots$ then $d_{H}\left(S_{N_{i}}, S_{N_{i+1}}\right)<\frac{\varepsilon}{2^{i}}$ since $N_{i+1}>N_{i}$,

$\Rightarrow$ there exists a sequence $s_{N_{i}} \in S_{N_{i}}, i \geq 1$ such that $\left|s_{N_{i}}-s_{N_{i+1}}\right|<\frac{\varepsilon}{2^{i}}$ and $S_{N_{i+1}} \in S_{N_{i+1}}$.

Let's show that the sequence $s_{N_{k}}$ is a Cauchy sequence.

Suppose that $m<n$ then

$$
\begin{aligned}
\left|s_{N_{m}}-s_{N_{n}}\right| & =\left|s_{N_{m}}-s_{N_{m+1}}+s_{N_{m+1}}-s_{N_{m+2}}+\cdots+s_{N_{n-1}}-s_{N_{n}}\right| \\
& <\left|s_{N_{m}}-s_{N_{m+1}}\right|+\left|s_{N_{m+1}}-s_{N_{m+2}}\right|+\cdots+\left|s_{N_{n-1}}-s_{N_{n}}\right| \\
& <\frac{\varepsilon}{2^{m}}+\frac{\varepsilon}{2^{m+1}}+\frac{\varepsilon}{2^{m+2}}+\cdots+\frac{\varepsilon}{2^{n-1}} \\
& <\varepsilon\left(\frac{1}{2^{m}}+\frac{1}{2^{m+1}}+\cdots+\frac{1}{2^{n-1}}\right) \\
& <\varepsilon\left(\frac{1}{2}+\frac{1}{2^{2}}+\cdots\right)=\varepsilon
\end{aligned}
$$

$\Rightarrow s_{N_{k}}$ is a Cauchy sequence.

Since $s_{N_{k}}$ is a sequence of real numbers therefore $\lim _{k \rightarrow \infty} S_{N_{k}}=L$ exists.

Let $S$ be the set of those limits that is

$$
S=\left\{L / L=\lim _{k \rightarrow \infty} S_{N_{k}}, s_{N_{k}} \in S_{N_{k}}, N_{1}<N_{2}<\cdots\right\}
$$

Figure 1 shows a subsequence $S_{N_{k}}$ of sets from $S_{N}$. Several subsequences of real numbers $a_{N_{k}}, b_{N_{k}}, \cdots$ are depicted.

Now, let's show that $\lim _{n \rightarrow \infty} S_{n}=S$ that is $\forall \varepsilon>0 \quad \exists K(\varepsilon) \in \mathbb{N} \quad$ such that if $n \geq K(\varepsilon)$ then $d_{H}\left(S_{n}, S\right)<\varepsilon$ that is $S_{n} \subset N_{\varepsilon}(S)$ and $S \subset N_{\varepsilon}\left(S_{n}\right)$.

First let's start by showing that $S \subset N_{\varepsilon}\left(S_{n}\right)$. 


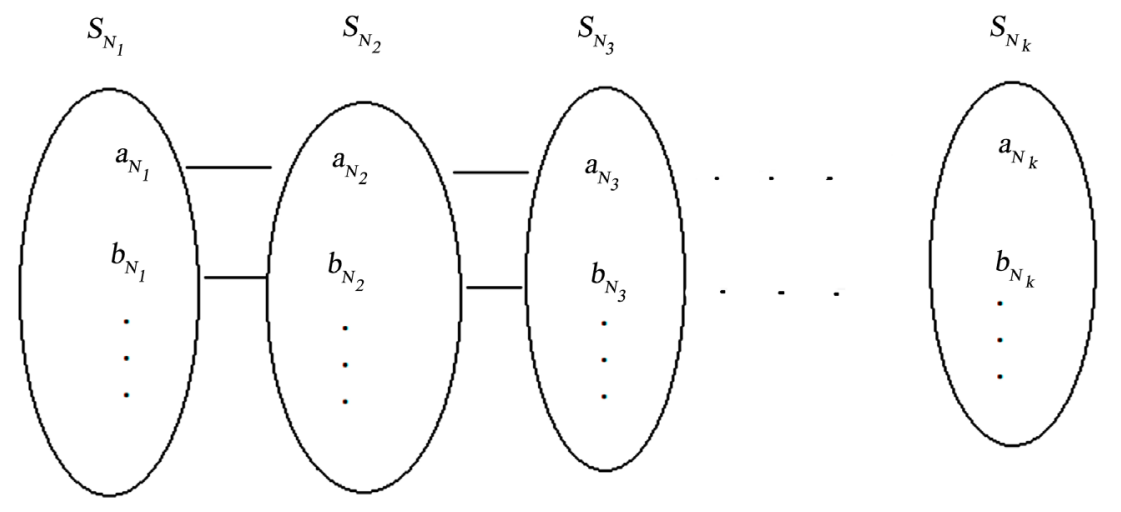

Figure 1. Subsequence $S_{N_{k}}$ of sets from $S_{n}$.

Let $s \in S \Rightarrow \exists s_{N_{k}} \in S_{N_{k}}$ such that $\lim _{k \rightarrow \infty} s_{N_{k}}=s \Rightarrow \forall \varepsilon>0 \quad \exists P(\varepsilon) \in \mathbb{N}$ such that if $K(\varepsilon) \geq P(\varepsilon)$ then $\left|s_{N_{k}}-s\right|<\frac{\varepsilon}{2}$.

Since $S_{n}$ is a Cauchy sequence then $\forall \varepsilon>0 \quad \exists M(\varepsilon) \in \mathbb{N}$ such that if $n, m \geq M(\varepsilon)$ then $d_{H}\left(S_{n}, S_{m}\right)<\frac{\varepsilon}{2}$ which means that $S_{n} \subset N_{\frac{\varepsilon}{2}}\left(S_{m}\right)$ and $S_{m} \subset N_{\frac{\varepsilon}{2}}\left(S_{n}\right) \quad n \geq M(\varepsilon)$.

Assuming that $N_{k} \geq M(\varepsilon), n \geq M(\varepsilon)$ then $d_{H}\left(S_{n}, S_{N_{k}}\right)<\frac{\varepsilon}{2}$.

$\Rightarrow$ there exists a sequence $s_{n} \in S_{n}$ such that $\left|s_{n}-s_{N_{k}}\right|<\frac{\varepsilon}{2}$.

Now let $L(\varepsilon)$ be the maximum of $P(\varepsilon)$ and $M(\varepsilon)$.

If $n, k>L(\varepsilon)$ then $\left|s-s_{n}\right| \leq\left|s-s_{N_{k}}\right|+\left|s_{N_{k}}-s_{n}\right|<\frac{\varepsilon}{2}+\frac{\varepsilon}{2}=\varepsilon$

$$
\Rightarrow s \in N_{\varepsilon}\left(A_{n}\right) \Rightarrow S \subset N_{\varepsilon}\left(S_{n}\right)
$$

Now, let's show that $S_{n} \subset N_{\varepsilon}(S) \quad \forall n \geq K(\varepsilon)$ for some $K(\varepsilon) \in \mathbb{N}$.

Let $b \in S_{n}$, since $S_{n}$ is a Cauchy sequence then $\forall \varepsilon_{i}=\frac{\varepsilon}{2^{i}}>0 \quad \exists N_{i}(\varepsilon) \in \mathbb{N}$ Such that if $n, m \geq N_{i}(\varepsilon)$ then $d_{H}\left(S_{n}, S_{m}\right)<\frac{\varepsilon}{2^{i}}, i \geq 1$.

If $b \in A_{n}$ for some $n \geq N_{i}(\varepsilon)$ then $\exists l \in \mathbb{N}$ such that $b=b_{N_{l}}$ where $b_{N_{l}} \in A_{N_{l}}$.

Since $\lim _{k \rightarrow \infty} b_{N_{k}}=s$ where $b \in S$, then for $k>l$,

$$
\begin{aligned}
\left|b_{N_{l}}-b_{N_{k}}\right| & \leq\left|b_{N_{l}}-b_{N_{l+1}}\right|+\left|b_{N_{l+1}}-b_{N_{l+2}}\right|+\cdots+\left|b_{N_{k-1}}-b_{N_{k}}\right| \\
& <\frac{\varepsilon}{2^{l}}+\frac{\varepsilon}{2^{l+1}}+\cdots+\frac{\varepsilon}{2^{k-1}} \\
& <\varepsilon\left(\frac{1}{2^{l}}+\frac{1}{2^{l+1}}+\cdots+\frac{1}{2^{k-1}}\right) \\
& <\varepsilon\left(\frac{1}{2}+\frac{1}{2^{2}}+\frac{1}{2^{3}}+\cdots\right)=\varepsilon \\
\Rightarrow \lim _{k \rightarrow \infty} \mid b_{N_{l}} & -b_{N_{k}}|\leq \varepsilon \Rightarrow| b_{N_{l}}-s|\leq \varepsilon \Rightarrow| b-s \mid \leq \varepsilon \Rightarrow b \in N_{\varepsilon}(S) \Rightarrow S_{n} \subset N_{\varepsilon} \\
\Rightarrow \lim _{n \rightarrow \infty} S_{n} & =S .
\end{aligned}
$$


Let's show that the set $S$ is a compact set that is bounded and closed set.

Let's start showing that $S$ is closed. let $x_{n}$ be a sequence in $S$ such that $\lim _{n \rightarrow \infty} x_{n}=x$.

Let's show that $x \in S$.

$\lim _{n \rightarrow \infty} x_{n}=x \Leftrightarrow \forall \varepsilon>0 \quad \exists N(\varepsilon) \in \mathbb{N}$ such that if $n \geq N(\varepsilon)$ then $\left|x_{n}-x\right|<\frac{\varepsilon}{2}$.

Since $x_{n} \in S \Rightarrow \exists$ a sequence $\left(x_{n}\right)_{N_{k}} \in S_{N_{k}}$ such that $\lim _{k \rightarrow \infty}\left(x_{n}\right)_{N_{k}}=x_{n}$.

$\Rightarrow \forall \varepsilon>0 \quad \exists M(\varepsilon) \in \mathbb{N}$ such that if $k \geq M(\varepsilon)$ then $\left|\left(x_{n}\right)_{N_{k}}-x_{n}\right|<\frac{\varepsilon}{2}$.

Assuming that $n \geq N(\varepsilon)$ and $k \geq M(\varepsilon)$ then

$\left|\left(x_{n}\right)_{N_{k}}-x\right| \leq\left|\left(x_{n}\right)_{N_{k}}-x_{n}\right|+\left|x_{n}-x\right|<\frac{\varepsilon}{2}+\frac{\varepsilon}{2}=\varepsilon$.

$\rightarrow \lim _{n \rightarrow \infty}\left(x_{n}\right)_{N_{k}}=x \Rightarrow x \in S$ therefore $S$ is closed.

Let's show that the set $S$ is a bounded set.

Since $\lim _{n \rightarrow \infty} S_{n}=S \Rightarrow$ for $\varepsilon=1 \quad \exists N(\varepsilon) \in \mathbb{N}$ such that $d_{H}\left(S_{n}, S\right)<\varepsilon$ $\rightarrow S \subset N_{1}\left(S_{n}\right)$ for $n \geq N(\varepsilon)$.

Since $S_{n}$ is bounded then $A_{n} \subset \bar{B}_{r}(x)$ for some $x \in S_{n}$ and some $r>0$, where $\bar{B}_{r}(x)=\{y \in \mathbb{R} /|y-x| \leq r\}$ then $N_{1}\left(A_{n}\right) \subset \bar{B}_{r+1}(x) \Rightarrow S \subset \bar{B}_{r+1}(x)$.

Therefore $S$ is bounded.

- The function $f$ defined as $f(X)=\bigcup_{1 \leq i \leq m} C_{i}(X)$ is a contraction using the Hausdorff metric.

So, we need to show that $d_{H}(f(X), f(Y))<r d_{H}(X, Y)$ where $r<1$.

$$
d_{H}(f(X), f(Y))=d_{H}\left(\bigcup_{1 \leq i \leq m} C_{i}(X), \bigcup_{1 \leq i \leq m} C_{i}(Y)\right) \text {. }
$$

Let's show that if $\max _{1 \leq i \leq m} d_{H}\left(C_{i}(X), C_{i}(Y)\right)<l$ then

$d_{H}\left(\bigcup_{1 \leq i \leq m} C_{i}(X), \bigcup_{1 \leq i \leq m} C_{i}(Y)\right)<l$.

Suppose that $\max d_{H}\left(C_{i}(X), C_{i}(Y)\right)<l$ then $d_{H}\left(C_{i}(X), C_{i}(Y)\right)<l$ for all $i \Rightarrow C_{i}(X) \subset N_{l}\left(C_{i}(Y)\right)$ and $C_{i}(Y) \subset N_{l}\left(C_{i}(X)\right)$ for all $i$.

$$
C_{i}(X) \subset N_{l}\left(C_{i}(Y)\right) \Rightarrow \bigcup_{1 \leq i \leq m} C_{i}(X) \subset \bigcup_{1 \leq i \leq m} N_{l}\left(C_{i}(Y)\right)
$$

Let's show that $\bigcup_{1 \leq i \leq m} N_{l}\left(C_{i}(Y)\right)=N_{l}\left(\bigcup_{1 \leq i \leq m} C_{i}(Y)\right)$.

Let $x \in \bigcup_{1 \leq i \leq m} N_{l}\left(C_{i}(Y)\right) \Leftrightarrow \exists i$ such that $x \in N_{l}\left(C_{i}(Y)\right) \Leftrightarrow|x-y|<l$ for some $y \in C_{i}(Y)$.

Then for some

$$
\begin{aligned}
& y \in \bigcup_{1 \leq i \leq m} C_{i}(Y) \Leftrightarrow x \in N_{l}\left(\bigcup_{1 \leq i \leq m} C_{i}(Y)\right) \\
& \Rightarrow \bigcup_{1 \leq i \leq m} N_{l}\left(C_{i}(Y)\right)=N_{l}\left(\bigcup_{1 \leq i \leq m} C_{i}(Y)\right) \\
& \Rightarrow \bigcup_{1 \leq i \leq m} C_{i}(X) \subset N_{l}\left(\bigcup_{1 \leq i \leq m} C_{i}(Y)\right) .
\end{aligned}
$$


Same thing, we can show that

$$
\bigcup_{1 \leq i \leq m} C_{i}(Y) \subset N_{l}\left(\bigcup_{1 \leq i \leq m} C_{i}(X)\right) .
$$

Therefore

$$
\begin{aligned}
& d_{H}\left(\bigcup_{1 \leq i \leq m} C_{i}(X), \bigcup_{1 \leq i \leq m} C_{i}(Y)\right)<l \\
& \Rightarrow d_{H}\left(\bigcup_{1 \leq i \leq m} C_{i}(X), \bigcup_{1 \leq i \leq m} C_{i}(Y)\right) \leq \max _{1 \leq i \leq m} d_{H}\left(C_{i}(X), C_{i}(Y)\right)
\end{aligned}
$$

Since $C_{i}$ is a contraction then $d_{H}\left(C_{i}(x), C_{i}(Y)\right)<r_{i} d_{H}(X, Y), \quad 0<r_{i}<1$.

$$
\Rightarrow d_{H}\left(\bigcup_{1 \leq i \leq m} C_{i}(X), \bigcup_{1 \leq i \leq m} c_{i}(Y)\right) \leq \underset{1 \leq i \leq m}{c} d_{H}(X, Y)
$$

where $c=\max _{1 \leq i \leq m} r_{i}$ so $0<c<1$.

Therefore $f$ is a contraction. Since $\mathcal{C}$ is a complete space and $f$ is a contraction, then according to the Banach's Contraction Mapping Theorem, we conclude that $f$ has a fix point, that is there exists $F$ such $f(F)=F$ therefore $F=\bigcup_{i=1}^{m} C_{i}(F)$ has a solution.

We can also prove that $F=\bigcap_{i=0}^{\infty} f^{i}(X)$ where $X \in \mathcal{C}$.

$\lim _{k \rightarrow \infty} f^{k}(X)=f\left(\lim _{k \rightarrow \infty} f^{k-1}(X)\right)$ since $f$ is continuous because $f$ is a contraction.

$$
\Rightarrow \lim _{k \rightarrow \infty} f^{k}(X)=F \text {. }
$$

Since $f(X) \subset X$ because $f$ is a contraction, therefore $\left\{f^{k}(X), k \geq 0\right\}$ are nested sets $\Rightarrow \lim _{k \rightarrow \infty} f^{k}(X)=\bigcap_{k \geq 0} f^{k}(X) \Rightarrow \bigcap_{k \geq 0} f^{k}(X)=F$.

Figure 2 below shows the successive iterations of $f$ defined as $f(X)=\bigcup_{i=1}^{k} C_{i}(X)$ starting with the initial compact set $F_{0}$.

By induction, we obtain

$$
f^{n}\left(F_{0}\right)=\bigcup_{i=1, j=1, \cdots, l=1}^{k} C_{i}\left(C_{j}\left(\cdots C_{l}\left(F_{0}\right)\right)\right)
$$

So we can define the fractal $F_{\infty}$ associated to the function $f$ and $F_{0}$ as the subset of $F_{0}$ defined by

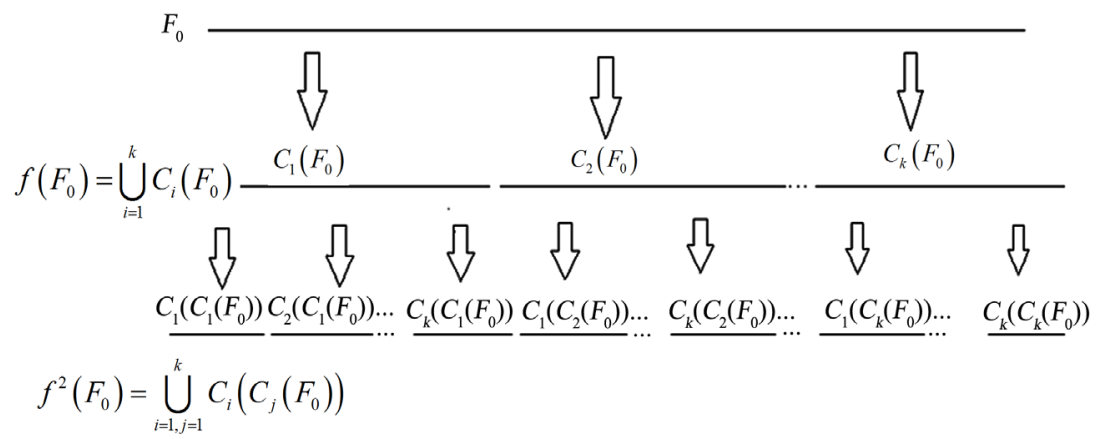

Figure 2. Successive iteration of $f$ starting with the initial compact $F_{0}$. 


$$
F_{\infty}=\bigcap_{n=0}^{\infty} f^{n}\left(F_{0}\right)=F_{0} \cap f\left(F_{0}\right) \cap f\left(f\left(F_{0}\right)\right) \cap f\left(f\left(f\left(F_{0}\right)\right)\right) \cap \cdots
$$

The next example is an example of fractals that can be obtained using IFS.

Example 1. (Cantor Set)

Starting with $K_{0}=[0,1]$, we obtain the subsequent subsets by acting two contractions with the same scale factor of $\frac{1}{3}: f_{1}(x)=\frac{1}{3} x$ and $f_{2}(x)=1-\frac{1}{3} x$ on $K_{0}=[0,1]$, then we get $K_{1}$. After repeating this process recursively in the contracted intervals, we obtain the Cantor set $K_{\infty}$ as shown Figure 3 below.

And then we can see that: $K_{\infty}=\bigcup_{i=1}^{2} f_{i}\left(K_{\infty}\right)$.

\section{The Box-Counting Dimension}

We know that from our Euclidean understanding of dimensions, a point has zero dimension, a line segment has one dimension, a square has two dimensions, and a cube has three dimensions. We also know that this understanding comes from our designation of the number of parameters we use to locate points and measure distance within given space.

Now, we use Hausdorff dimension [11] when we deal with a non-Euclidean space for instance fractals. The Hausdorff dimension is more sophisticated.

Fortunately, when the fractal arises from contractions, we have the following theorem that gives a way to estimate the Hausdorff dimension easily.

Theorem 2. Let $\left\{C_{1}, C_{2}, \cdots, C_{n}\right\}$ be an iterated function system (IFS) with $n \geq 2$. Let $r_{1}, r_{2}, \cdots, r_{n}$ be respectively the scales of the contractions.

If $F_{\infty}$ is the attractor of the IFS, then the Hausdorff dimension $d$ of $F_{\infty}$ satisfies the equation:

$$
\sum_{i=1}^{n} r_{i}^{d}=1
$$

the equation: $\sum_{i=1}^{n} r_{i}^{d}=1$ is known as the Moran Equation.

With the Moran equation, we can approximate the dimension of a fractal obtained using multiple contractions.

Another way of computing the dimension of a fractal is through the box counting method I describe below.

Let $S$ be a bounded $d$-dimensional set. Imagine covering $S$ by $d$-dimensional cubes of side length $\varepsilon$.

Let $N(\varepsilon)$ be the number of cubes of side length $\varepsilon$ needed to cover $S$.

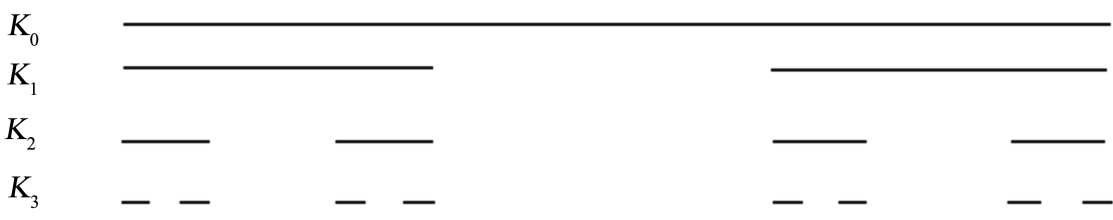

$K_{\infty}$

Figure 3. Cantor set. 
If $d=1$. So we can consider line segments of length $\varepsilon . N(\varepsilon) \approx \frac{C}{\varepsilon}$ where $C$ is a constant. The exponent 1 of $\varepsilon$ represents the dimension of the line segment.

If $d=2$, we can consider squares of side $\varepsilon$ then $N(\varepsilon) \approx \frac{C}{\varepsilon^{2}}$. The exponent 2 of $\varepsilon$ represents the dimension of the square.

If $d=3$, then we can consider cubes of side $\varepsilon$ and then $N(\varepsilon) \approx \frac{C}{\varepsilon^{3}}$. The exponent 3 of $\varepsilon$ represents the dimension of the cube.

We conclude that for a bounded d-dimensional set, $N(\varepsilon) \approx \frac{C}{\varepsilon^{d}}$. To find the general formula for the dimension of a given bounded shape whether it is an Euclidean shape or a fractal shape we can solve the equation:

$$
\begin{gathered}
N(\varepsilon) \approx \frac{C}{\varepsilon^{d}} \\
\ln (N(\varepsilon)) \approx \ln \left(\frac{C}{\varepsilon^{d}}\right) \\
d \approx \frac{\ln (N(\varepsilon))}{\ln \left(\frac{1}{\varepsilon}\right)}-\frac{\ln (C)}{\ln \left(\frac{1}{\varepsilon}\right)}
\end{gathered}
$$

When $\varepsilon$ is very small that is when $\varepsilon$ approaches 0 then

$$
\lim _{\varepsilon \rightarrow 0^{+}}\left(\frac{\ln (N(\varepsilon))}{\ln \left(\frac{1}{\varepsilon}\right)}-\frac{\ln (C)}{\ln \left(\frac{1}{\varepsilon}\right)}\right)=\lim _{\varepsilon \rightarrow 0^{+}} \frac{\ln (N(\varepsilon))}{\ln \left(\frac{1}{\varepsilon}\right)}
$$

which leads to the definition of box-counting dimension.

Definition 3. The box counting dimension denoted boxdim $(S)$ of a bounded set $S$ is given by:

$$
\operatorname{boxdim}(S)=\lim _{\varepsilon \rightarrow 0^{+}} \frac{\ln (N(\varepsilon))}{\ln \left(\frac{1}{\varepsilon}\right)}
$$

We can easily show that the box-counting dimension agrees with the standard notion of dimension see [1]. Now let's provide some simplifications to this definition.

Proposition 1. Let $S$ be a bounded set of $\mathbb{R}^{m}$.

For all $\varepsilon \in(0,1), \exists b_{n}$ such that $b_{n+1}<\varepsilon<b_{n}$ and $\lim _{n \rightarrow \infty} b_{n}=0$ and $\lim _{n \rightarrow \infty} \frac{\ln \left(b_{n+1}\right)}{\ln \left(b_{n}\right)}=1$.

Proof. For any postive real number $a$, there exists $n$ such that $n \leq a \leq n+1$ in particular when $a=\frac{\ln \left(\frac{1}{\varepsilon}\right)}{\ln 2}>0$. Therefore we conclude that

$$
\ln 2^{-(n+1)} \leq \ln (\varepsilon) \leq \ln 2^{-n}
$$




$$
2^{-(n+1)} \leq \varepsilon \leq 2^{-n}
$$

So we can choose $b_{n}=2^{-n}$ then $\lim _{n \rightarrow \infty} b_{n}=0$ and $\lim _{n \rightarrow \infty} \frac{\ln \left(b_{n+1}\right)}{\ln \left(b_{n}\right)}=1$

Theorem 3. Assuming that $b_{1}>b_{2}>b_{3}>\cdots>b_{n}>\cdots$ and $\lim _{n \rightarrow \infty} b_{n}=0$ and $\lim _{n \rightarrow \infty} \frac{\ln \left(b_{n+1}\right)}{\ln \left(b_{n}\right)}=1$. If $\lim _{n \rightarrow \infty} \frac{\ln \left(N\left(b_{n}\right)\right)}{\ln \left(\frac{1}{b_{n}}\right)}=d$ then $\lim _{\varepsilon \rightarrow 0} \frac{\ln (N(\varepsilon))}{\ln \left(\frac{1}{\varepsilon}\right)}=d$

Proof. We start the proof with a bounded set $S$ in $\mathbb{R}^{2}$. Let $N(\varepsilon)$ be the number of boxes of side length $\varepsilon$ needed to cover $S$.

Let $S_{1}$ be any subset of $S$ being covered by a box of side length $b_{n+1}$.

We then can cover the subset $S_{1}$ by almost 4 boxes of side length $\varepsilon$. Therefore $N(\varepsilon) \leq 4 N\left(b_{n+1}\right)$. Since $\varepsilon \leq b_{n}$ then $N\left(b_{n}\right) \leq 4 N(\varepsilon)$. This implies that

$$
\frac{N\left(b_{n}\right)}{2^{2}} \leq N(\varepsilon) \leq 2^{2} N\left(b_{n+1}\right)
$$

And thus in $\mathbb{R}^{m}$ we obtain

$$
\frac{N\left(b_{n}\right)}{2^{m}} \leq N(\varepsilon) \leq 2^{m} N\left(b_{n+1}\right)
$$

Since $\ln$ is an increasing function then

$$
-m \ln (2)+\ln \left(N\left(b_{n}\right)\right) \leq \ln (N(\varepsilon)) \leq m \ln (2)+\ln \left(N\left(b_{n+1}\right)\right)
$$

Now using the fact that

$$
b_{n+1} \leq \varepsilon \leq b_{n}
$$

we obtain the following inequality

$$
\frac{\ln \left(\frac{1}{b_{n}}\right)}{\ln \left(\frac{1}{b_{n+1}}\right)} \frac{-m \ln (2)+\ln \left(N\left(b_{n}\right)\right)}{\ln \left(\frac{1}{b_{n}}\right)} \leq \frac{\ln (N(\varepsilon))}{\ln \left(\frac{1}{\varepsilon}\right)} \leq \frac{\ln \left(\frac{1}{b_{n+1}}\right)}{\ln \left(\frac{1}{b_{n}}\right)} \frac{m \ln (2)+\ln \left(N\left(b_{n+1}\right)\right)}{\ln \left(\frac{1}{b_{n+1}}\right)}
$$

Now using the squeeze theorem, we see that if $\lim _{n \rightarrow \infty} \frac{\ln \left(N\left(b_{n}\right)\right)}{\ln \left(\frac{1}{b_{n}}\right)}=d$ then $\lim _{\varepsilon \rightarrow 0^{+}} \frac{\ln (N(\varepsilon))}{\ln \left(\frac{1}{\varepsilon}\right)}=d \quad$ therefore the boxdim $=\mathrm{d}$.

There are other simplifications we can use to get the dimension of the fractals. We can replace $N(\varepsilon)$ by $N_{0}(\varepsilon)$ which is the smallest number of boxes needed to cover the set. Also, instead of using squares, we can use disks of radius $\varepsilon$ or triangles of side length $\varepsilon$.

Example 2. Let's find the box counting dimension of the Cantor set. From the figure in example 1 , the length of each interval of $K_{1}$ equals $\frac{1}{3}$ so $b_{1}=\frac{1}{3}$. 
The number of boxes needed to cover these intervals is 2 so $N\left(b_{1}\right)=2$.

Step 2, the length of each interval of $K_{2}$ equals $\frac{1}{3^{2}}$ so $b_{2}=\frac{1}{3^{2}}$. The number of boxes needed to cover these intervals is $2^{2}$ so $N\left(b_{2}\right)=2^{2}$.

Continuing until the nth step, be see that $b_{n}=\frac{1}{3^{n}}$ and $N\left(b_{n}\right)=2^{n}$

$$
\operatorname{boxdim}(K)=\lim _{n \rightarrow \infty} \frac{\ln \left(2^{n}\right)}{\ln \left(3^{n}\right)}=\frac{\ln (2)}{\ln (3)}
$$

\section{Box-Counting Dimension for Fractals That Arise from Multitude of Scalings}

\subsection{The Case of a One Dimensional Set}

Theorem 4. Let $K_{0}$ be a bounded set of dimension 1 .

Let $C_{i}, 1 \leq i \leq k$ be contractions on $K_{0}$ with corresponding scale factors $r_{i}=\frac{a_{i}}{b_{i}}, 1 \leq i \leq k$ where $a_{i}$ and $b_{i}$ are both integers.

Let $r$ be the largest rational number such that $\frac{r_{i}}{r}$ is an integer for all $1 \leq i \leq k$ that is $r=\frac{\operatorname{gcd}\left(a_{i}\right)}{\operatorname{lcm}\left(b_{i}\right)}, 1 \leq i \leq k$.

If $K_{\infty}$ is the fractal that results from $K_{0}$ using the contractions $C_{1}, C_{2}, C_{3}, \cdots, C_{k}$ then

$$
\operatorname{boxdim}\left(K_{\infty}\right)=-\frac{\ln \left(\frac{r_{1}}{r}+\frac{r_{2}}{r}+\frac{r_{3}}{r}+\cdots+\frac{r_{k}}{r}\right)}{\ln (r)}
$$

Proof. Consider a set of dimension one for instance the segment $K_{0}=[0,1]$. Let $K_{1}$ be the set obtained by acting $k$ contractions with scales factors $r_{1}, r_{2}, \cdots, r_{k}$ on the segment $K_{0}=[0,1]$. For the next step, let $K_{2}$ be the set obtained by acting the $n$ contractions on each interval of the set $K_{1}$. Repeating this process, we obtain an attractor denoted $K_{\infty}$ as seen in Figure 4 below. $K_{\infty}$ is a fractal.

Let's find the dimension of the fractal $K_{\infty}$. Let $r$ be the largest rational number such that $\frac{r_{i}}{r}$ is an integer for all $1 \leq i \leq k$ that is $r=\frac{\operatorname{gcd}\left(a_{i}\right)}{\operatorname{lcm}\left(b_{i}\right)}$.

Define $b_{1}=r$ and let $N\left(b_{1}\right)$ be the number of intervals of length $b_{1}$ needed to cover $K_{1}$ so

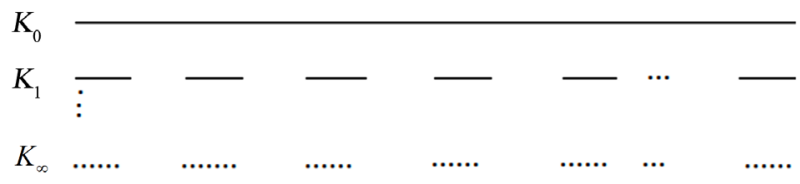

Figure 4. Fractal from one dimensional that arises from a multitude of scaling. 


$$
N\left(b_{1}\right)=\frac{r_{1}}{r}+\frac{r_{2}}{r}+\frac{r_{3}}{r}+\cdots+\frac{r_{k}}{r}
$$

For the second step, define $b_{2}=r^{2}$ and let $N\left(b_{2}\right)$ be the number of intervals of length $b_{2}$ needed to cover $K_{2}$. So

$$
\begin{aligned}
N\left(b_{2}\right)= & \left(\frac{r_{1}^{2}}{r^{2}}+\frac{r_{1} r_{2}}{r^{2}}+\frac{r_{1} r_{3}}{r^{2}}+\cdots+\frac{r_{1} r_{k}}{r^{2}}\right)+\left(\frac{r_{1} r_{2}}{r^{2}}+\frac{r_{2}^{2}}{r^{2}}+\frac{r_{2} r_{3}}{r^{2}}+\frac{r_{2} r_{4}}{r^{2}}+\cdots+\frac{r_{2} r_{k}}{r^{2}}\right) \\
& +\left(\frac{r_{1} r_{3}}{r^{2}}+\frac{r_{2} r_{3}}{r^{2}}+\frac{r_{3}^{2}}{r^{2}}+\frac{r_{3} r_{4}}{r^{2}}+\cdots+\frac{r_{3} r_{k}}{r^{2}}\right)+\cdots \\
& +\left(\frac{r_{1} r_{k}}{r^{2}}+\frac{r_{2} r_{k}}{r^{2}}+\frac{r_{3} r_{k}}{r^{2}}+\frac{r_{4} r_{k}}{r^{2}}+\cdots+\frac{r_{k}^{2}}{r^{2}}\right) \\
= & \frac{r_{1}}{r}\left(\frac{r_{1}}{r}+\frac{r_{2}}{r}+\frac{r_{3}}{r}+\cdots+\frac{r_{k}}{r}\right)+\frac{r_{2}}{r}\left(\frac{r_{1}}{r}+\frac{r_{2}}{r}+\frac{r_{3}}{r}+\cdots+\frac{r_{k}}{r}\right) \\
& +\frac{r_{3}}{r}\left(\frac{r_{1}}{r}+\frac{r_{2}}{r}+\frac{r_{3}}{r}+\cdots+\frac{r_{k}}{r}\right)+\cdots+\frac{r_{k}}{r}\left(\frac{r_{1}}{r}+\frac{r_{2}}{r}+\frac{r_{3}}{r}+\cdots+\frac{r_{k}}{r}\right) \\
& N\left(b_{2}\right)=\left(\frac{r_{1}}{r}+\frac{r_{2}}{r}+\frac{r_{3}}{r}+\cdots+\frac{r_{k}}{r}\right)^{2}
\end{aligned}
$$

For the third step,

$$
b_{3}=r^{3} \text { and } N\left(b_{3}\right)=\left(\frac{r_{1}}{r}+\frac{r_{2}}{r}+\frac{r_{3}}{r}+\cdots+\frac{r_{k}}{r}\right)^{3}
$$

By induction,

$$
b_{n}=r^{n} \text { and } N\left(b_{n}\right)=\left(\frac{r_{1}}{r}+\frac{r_{2}}{r}+\frac{r_{3}}{r}+\cdots+\frac{r_{k}}{r}\right)^{n}
$$

Now, we can evaluate the boxdim of $K_{\infty}$ :

$$
\begin{gathered}
\operatorname{boxdim}\left(K_{\infty}\right)=\lim _{n \rightarrow \infty} \frac{\ln \left(N\left(b_{n}\right)\right)}{\ln \left(\frac{1}{b_{n}}\right)} \\
\operatorname{boxdim}\left(K_{\infty}\right)=-\frac{\ln \left(\frac{r_{1}}{r}+\frac{r_{2}}{r}+\frac{r_{3}}{r}+\cdots+\frac{r_{k}}{r}\right)}{\ln (r)}
\end{gathered}
$$

Remark 2. This formula could be also seen as the generalization of the Cantor set dimension in the case we apply a multitude of scales on the unit interval.

Example 3. If $r_{1}=\frac{2}{5}, r_{2}=\frac{1}{3}, r_{3}=\frac{1}{6}$ then $r=\frac{\operatorname{GCD}(2,1,1)}{\operatorname{LCM}(5,3,6)}=\frac{1}{30}$ therefore $\operatorname{boxdim}\left(K_{\infty}\right)=-\frac{\ln (12+10+5)}{\ln \left(\frac{1}{30}\right)}=\frac{\ln (27)}{\ln (30)} \approx 0.97$.

Remark 3. Using the Moran equation $\left(\frac{2}{5}\right)^{d}+\left(\frac{1}{3}\right)^{d}+\left(\frac{1}{6}\right)^{d}=1$, we would find the Haausdorff dimension $d \approx 0.92$ which is very close to the boxplot dimension. 


\subsection{The Case of a Bounded Set of Any Arbitrary Dimension}

Theorem 5. Let $K_{0}$ be a bounded set of dimension $m$.

Let $C_{1}, C_{2}, C_{3}, \cdots, C_{k}$ be contractions on $K_{0}$ with corresponding scale factors $r_{i}=\frac{a_{i}}{b_{i}}, 1 \leq i \leq k$ where $a_{i}$ and $b_{i}$ are both integers.

Let $r$ be the largest rational number such that $\frac{r_{i}}{r}$ is an integer for all $1 \leq i \leq k$ that is $r=\frac{\operatorname{gcd}\left(a_{i}\right)}{\operatorname{lcm}\left(b_{i}\right)}, 1 \leq i \leq k$.

If $K_{\infty}$ is the fractal that results from $K_{0}$ using the contractions $C_{1}, C_{2}, C_{3}, \cdots, C_{k}$ then

$$
\operatorname{boxdim}\left(K_{\infty}\right)=-\frac{\ln \left(\left(\frac{r_{1}}{r}\right)^{m}+\left(\frac{r_{2}}{r}\right)^{m}+\left(\frac{r_{3}}{r}\right)^{m}+\cdots+\left(\frac{r_{k}}{r}\right)^{m}\right)}{\ln (r)}
$$

Proof. Starting with a bounded set $K_{0}$ in $\mathbb{R}^{2}$.

Let $K_{1}$ be the set obtained after acting a set of contractions $C_{1}, C_{2}, \cdots, C_{k}$ with scales factors $r_{1}, r_{2}, r_{3}, \cdots, r_{k}$ on the set $K_{0}$. Repeating the same process indefinitively, we obtain the limiting set denoted $K_{\infty}$ as shown in Figure 5 (using $r_{1}=\frac{1}{3}, r_{2}=\frac{1}{4}, r_{3}=\frac{1}{5}, r_{4}=\frac{1}{6}, r_{5}=\frac{1}{7}$ ).

$K_{\infty}$ is a fractal.

To find the dimension of $K_{\infty}$, we consider $B$ the smallest box of side length $I$ to cover $K_{0}$. Let $r$ be the largest rational number such that $\frac{r_{i}}{r}$ is an integer for all $1 \leq i \leq k$.

So for the first step of the fractal construction, if $b_{1}=r l$, then the number of boxes needed to cover $K_{1}$ is given by

$$
\begin{gathered}
N\left(b_{1}\right)=\frac{\left(r_{1} l\right)^{2}}{(r l)^{2}}+\frac{\left(r_{2} l\right)^{2}}{(r l)^{2}}+\frac{\left(r_{3} l\right)^{2}}{(r l)^{2}}+\frac{\left(r_{4} l\right)^{2}}{(r l)^{2}}+\cdots+\frac{\left(r_{k} l\right)^{2}}{(r l)^{2}} \\
N\left(b_{1}\right)=\frac{\left(r_{1}\right)^{2}}{r^{2}}+\frac{\left(r_{2}\right)^{2}}{r^{2}}+\frac{\left(r_{3}\right)^{2}}{r^{2}}+\frac{\left(r_{4}\right)^{2}}{r^{2}}+\cdots+\frac{\left(r_{k}\right)^{2}}{r^{2}}
\end{gathered}
$$

For the second step of the fractal construction, if $b_{2}=r^{2} l$, then

$$
\begin{aligned}
N\left(b_{2}\right)= & \left(\frac{\left(r_{1}^{2} l\right)^{2}}{\left(r^{2} l\right)^{2}}+\frac{\left(r_{1} r_{2} l\right)^{2}}{\left(r^{2} l\right)^{2}}+\frac{\left(r_{1} r_{3} l\right)^{2}}{\left(r^{2} l\right)^{2}}+\frac{\left(r_{1} r_{4} l\right)^{2}}{\left(r^{2} l\right)^{2}}+\cdots+\frac{\left(r_{1} r_{k} l\right)^{2}}{\left(r^{2} l\right)^{2}}\right) \\
& +\left(\frac{\left(r_{1} r_{2} l\right)^{2}}{\left(r^{2} l\right)^{2}}+\frac{\left(r_{2}^{2} l\right)^{2}}{\left(r^{2} l\right)^{2}}+\frac{\left(r_{2} r_{3} l\right)^{2}}{\left(r^{2} l\right)^{2}}+\cdots+\frac{\left(r_{2} r_{k} l\right)^{2}}{\left(r^{2} l\right)^{2}}\right)+\cdots \\
& +\left(\frac{\left(r_{1} r_{k} l\right)^{2}}{\left(r^{2} l\right)^{2}}+\frac{\left(r_{2} r_{k} l\right)^{2}}{\left(r^{2} l\right)^{2}}+\frac{\left(r_{3} r_{k} l\right)^{2}}{\left(r^{2} l\right)^{2}}+\cdots+\frac{\left(r_{k} l\right)^{2}}{\left(r^{2} l\right)^{2}}\right)
\end{aligned}
$$




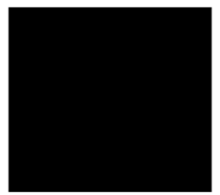

$K_{0}$

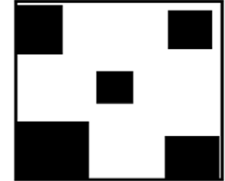

$K_{1}$

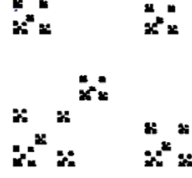

$K_{\mathrm{n}}$

Figure 5. Fractal from two dimension that arises from multiple scaling.

$$
\begin{aligned}
N\left(b_{2}\right)= & \frac{r_{1}^{2}}{r^{2}}\left(\frac{r_{1}^{2}}{r^{2}}+\frac{r_{2}^{2}}{r^{2}}+\frac{r_{3}^{2}}{r^{2}}+\cdots+\frac{r_{k}^{2}}{r^{2}}\right)+\frac{r_{2}^{2}}{r^{2}}\left(\frac{r_{1}^{2}}{r^{2}}+\frac{r_{2}^{2}}{r^{2}}+\frac{r_{3}^{2}}{r^{2}}+\cdots+\frac{r_{k}^{2}}{r^{2}}\right) \\
& +\cdots+\frac{r_{k}^{2}}{r^{2}}\left(\frac{r_{1}^{2}}{r^{2}}+\frac{r_{2}^{2}}{r^{2}}+\frac{r_{3}^{2}}{r^{2}}+\cdots+\frac{r_{k}^{2}}{r^{2}}\right)
\end{aligned}
$$

Therefore

$$
N\left(b_{2}\right)=\left(\frac{r_{1}^{2}}{r^{2}}+\frac{r_{2}^{2}}{r^{2}}+\frac{r_{3}^{2}}{r^{2}}+\cdots+\frac{r_{k}^{2}}{r^{2}}\right)^{2}
$$

By induction

$$
b_{n}=r^{n} l, \quad N\left(b_{n}\right)=\left(\frac{r_{1}^{2}}{r^{2}}+\frac{r_{2}^{2}}{r^{2}}+\frac{r_{3}^{2}}{r^{2}}+\cdots+\frac{r_{k}^{2}}{r^{2}}\right)^{n}
$$

The boxdim of $F_{\infty}$ is given by

$$
\operatorname{boxdim}\left(K_{\infty}\right)=-\frac{\ln \left(\frac{r_{1}^{2}}{r^{2}}+\frac{r_{2}^{2}}{r^{2}}+\frac{r_{3}^{2}}{r^{2}}+\cdots+\frac{r_{k}^{2}}{r^{2}}\right)}{\ln (r)}
$$

We conclude that in general that if $K_{0}$ is a bounded subset of $\mathbb{R}^{m}$ then the dimension of the fractal $K_{\infty}$ is given by

$$
\operatorname{boxdim}\left(K_{\infty}\right)=-\frac{\ln \left(\frac{r_{1}^{m}}{r^{m}}+\frac{r_{2}^{m}}{r^{m}}+\frac{r_{3}^{m}}{r^{m}}+\cdots+\frac{r_{k}^{m}}{r^{m}}\right)}{\ln (r)}
$$

Corollary 1. If $r_{1}=r_{2}=\cdots=r_{k}$ then the box counting dimension and the Hausdorf dimension of $F_{\infty}$ are both the same.

Proof. If $r_{1}=r_{2}=\cdots=r_{k}$ then The boxdim of $F_{\infty}$ is $-\frac{\ln (k)}{\ln \left(r_{1}\right)}$.

The Hausdorf dimension $d$ is such that $\sum_{i=1}^{k} r_{i}^{d}=1$. Since $r_{1}=r_{2}=\cdots=r_{k}$ then $d=-\frac{\ln (k)}{\ln \left(r_{1}\right)}$

Corollary 2. If $\sum_{m=1}^{k} \frac{1}{\left(\sum_{i=1}^{k} \frac{r_{i}}{r}\right)^{\frac{\ln \left(r_{m}\right)}{\ln (r)}}}=1$ where $r$ be the largest rational number such that $\frac{r_{i}}{r}$ is an integer for all $1 \leq i \leq k$, then the boxdim and the Hausdorf dimension of $K_{\infty}$ agree. 
The proof is straightforward.

Example 4. Consider the special case with 2 scale factors $r_{1}$ and $r_{2}$ and $r_{2}=r_{1}^{2}$. The sum in the corollary 2 becomes

$$
\frac{1}{1+\frac{r_{2}}{r_{1}}}+\frac{1}{\left(1+\frac{r_{2}}{r_{1}}\right)^{\frac{\ln \left(r_{2}\right)}{\ln \left(r_{1}\right)}}}=1
$$

Since $r_{2}=r_{1}^{2}$ then $r_{1}=\frac{-1+\sqrt{5}}{2}$ and therefore $\frac{r_{1}}{r_{2}}=\frac{1}{r_{1}}=\frac{1+\sqrt{5}}{2}$ which is the golden ratio.

\section{Convoluting Fractals and Dimension}

Definition 4. (Convolution) Let $G_{\infty}$ and $H_{\infty}$ be two fractals that represent the limiting sets of a given bounded set $S_{0}$ of $\mathbb{R}^{2}$ with scale factors $r_{1}, r_{2}, r_{3}, \cdots, r_{p}$ and $s_{1}, s_{2}, s_{3}, \cdots, s_{q}$ respectively. If $g(X)=\bigcup_{1 \leq i \leq p} r_{i}(X)$ and $h(X)=\bigcup_{1 \leq i \leq q} s_{i}(X)$ where $X \in \mathcal{C}$, then the convolution of $G_{\infty}$ and $H_{\infty}$ denoted $G_{\infty} \star H_{\infty}$ is given by

$$
\left.G_{\infty} \star H_{\infty}=\bigcap_{i=0}^{\infty}(h \circ g)^{i}\left(S_{0}\right)=S_{0} \bigcap\left(h\left(g\left(S_{0}\right)\right)\right) \bigcap\left(h\left(g\left(h\left(g\left(S_{0}\right)\right)\right)\right)\right)\right) \cdots
$$

In short, the fractal $G_{\infty} \star H_{\infty}$ is constructed by acting alternatively the functions $g$ on $S_{0}$ and $h$ on $g\left(S_{0}\right)$ Ad infinitum.

Example 5. We suppose that $g$ is the function whose action consists of constructing a $\frac{1}{3}$-copy of the circle whose interior is white and a $\frac{1}{2}$-copy of the same circle. The action of the second function consists of constructing two $\frac{1}{2}$ -copies of the circle and two $\frac{1}{4}$-copies of the same circle. The first stage and second stage of the convoluted fractal are obtained as shown in Figure 6 below.

Next, we will explore the dimension of the fractal $G_{\infty} \star H_{\infty}$ and see how it is related to the dimensions of $G_{\infty}$ and $H_{\infty}$. We will show in the next theorem that it is simply a weighted average of the two fractal dimensions.

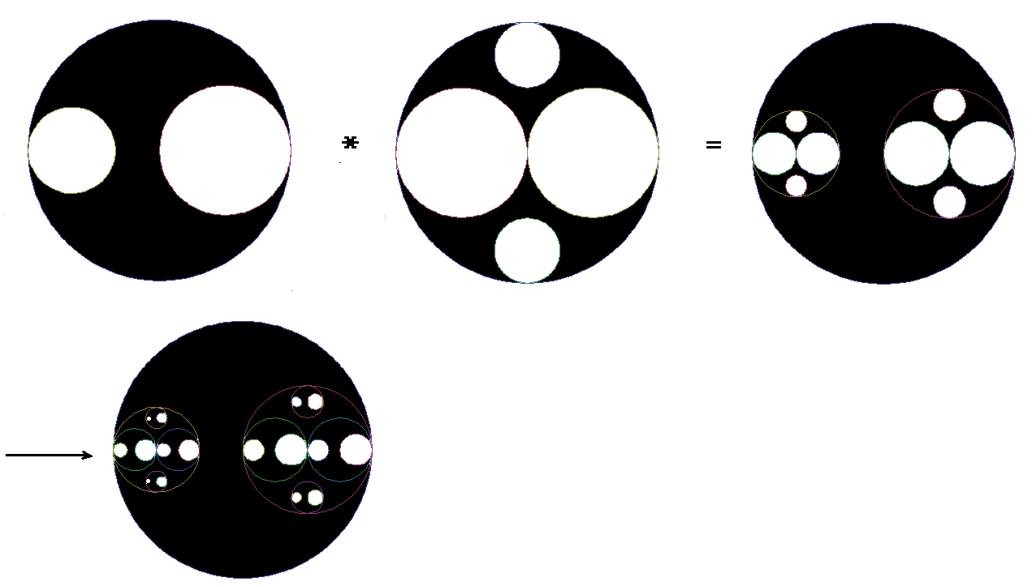

Figure 6. First two stages of the convoluted fractal. 
Theorem 6. If $d_{G_{\infty}}, d_{H_{\infty}}$ and $d_{G_{\infty} \star H_{\infty}}$ represent the box-counting dimension of $G_{\infty}, H_{\infty}$ and $G_{\infty} \star H_{\infty}$ respectively with $r$ be the largest rational number such that $\frac{r_{i}}{r}$ is an integer for all $1 \leq i \leq k$ and $s$ be the largest rational number such that $\frac{s_{i}}{S}$ is an integer for all $1 \leq i \leq k$, then $d_{G_{\infty} \star H_{\infty}}$ is given by the weighted average of $d_{G_{\infty}}$ and $d_{H_{\infty}}$ that is

$$
d_{G_{\infty} \star H_{\infty}}=\frac{d_{G_{\infty}}}{1+\frac{\ln (s)}{\ln (r)}}+\frac{d_{H_{\infty}}}{1+\frac{\ln (r)}{\ln (s)}}
$$

and if $d_{G_{\infty}} \leq d_{H_{\infty}}$ then $d_{G_{\infty}} \leq d_{G_{\infty} \star H_{\infty}} \leq d_{H_{\infty}}$.

Proof. It is easy to see that if $C$ and $T$ are both contractions defined respectively on the sets $S_{0}$ and $C\left(S_{0}\right)$ with scale factors respectively $r$ and $s$ then the composite $T \circ C$ is also a contraction with scale factor $r s$. Let $g(X)=\bigcup_{j=1}^{q} T_{j}(X)$ and $h(X)=\bigcup_{i=1}^{p} C_{i}(X)$.

Since $g \circ h\left(S_{0}\right)=\bigcup_{i=1}^{p} \bigcup_{j=1}^{q} T_{j}\left(C_{i}\left(S_{0}\right)\right)$ then $g \circ h$ is a function with $p q$ associated contractions $T_{j} \circ C_{i}, 1 \leq i \leq p, 1 \leq j \leq q$ whose scale factors are respectively $r_{i} s_{j}, 1 \leq i \leq p, 1 \leq j \leq q$. then according to the theorem 4 .

$$
\begin{aligned}
d_{G_{\infty} \times H_{\infty}}= & \frac{\ln \left(\left(\frac{r_{1} s_{1}}{r s}\right)^{m}+\left(\frac{r_{1} s_{2}}{r s}\right)^{m}+\left(\frac{r_{1} s_{3}}{r s}\right)^{m}+\cdots+\left(\frac{r_{1} s_{q}}{r s}\right)^{m}+\left(\frac{r_{2} s_{1}}{r s}\right)^{m}+\left(\frac{r_{2} s_{2}}{r s}\right)^{m}+\cdots+\left(\frac{r_{2} s_{q}}{r s}\right)^{m}+\cdots\right.}{\ln (r s)} \\
& +\frac{\left.\left(\frac{r_{p} s_{1}}{r s}\right)^{m}+\left(\frac{r_{p} s_{2}}{r s}\right)^{m}+\cdots+\left(\frac{r_{p} s_{q}}{r s}\right)^{m}\right)}{\ln (r s)}
\end{aligned}
$$

then

$$
\begin{gathered}
d_{G_{\infty} \star H_{\infty}}=\frac{\ln \left(\left(\frac{r_{1}}{r}\right)^{m}+\left(\frac{r_{2}}{r}\right)^{m}+\left(\frac{r_{3}}{r}\right)^{m}+\cdots+\left(\frac{r_{p}}{r}\right)^{m}\right)+\ln \left(\left(\frac{s_{1}}{s}\right)^{m}+\left(\frac{s_{2}}{s}\right)^{m}+\left(\frac{s_{3}}{s}\right)^{m}+\cdots+\left(\frac{s_{q}}{s}\right)^{m}\right)}{\ln (s)+\ln (r)} \\
d_{G_{\infty} \star H_{\infty}}=\frac{d_{G_{\infty}}}{1+\frac{\ln (s)}{\ln (r)}+\frac{d_{H_{\infty}}}{1+\frac{\ln (r)}{\ln (s)}}}
\end{gathered}
$$

Since $d_{G_{\infty}} \leq d_{H_{\infty}}$ then

$$
\frac{d_{G_{\infty}}}{1+\frac{\ln (s)}{\ln (r)}}+\frac{d_{G_{\infty}}}{1+\frac{\ln (r)}{\ln (s)}} \leq d_{G_{\infty} \star H_{\infty}} \leq \frac{d_{H_{\infty}}}{1+\frac{\ln (s)}{\ln (r)}}+\frac{d_{H_{\infty}}}{1+\frac{\ln (r)}{\ln (s)}}
$$

Therefore $d_{G_{\infty}} \leq d_{G_{\infty} \star H_{\infty}} \leq d_{H_{\infty}}$.

Example 6. From the previous picture,

$r_{1}=\frac{1}{3}, r_{2}=\frac{1}{2}, s_{1}=\frac{1}{4}, s_{2}=\frac{1}{4}, s_{3}=\frac{1}{2}, s_{4}=\frac{1}{2}$ then $r=\frac{1}{6}$ and $s=\frac{1}{4}$ therefore ac- 
cording to Equation (4) $d_{G_{\infty}}=\frac{\ln (13)}{\ln 6} \approx 1.43, d_{H_{\infty}}=\frac{\ln (10)}{\ln 4} \approx 1.66$, using Equation (6), we calculate the box-counting dimension of the convoluted fractal and find: $d_{G_{\infty} \star H_{\infty}}=1.53$.

All the corollaries from [7] follow easily.

Corollary 3. If $d_{G_{\infty}}=d_{H_{\infty}}$ then $d_{G_{\infty}}=d_{G_{\infty} \star H_{\infty}}=d_{H_{\infty}}$

Corollary 4. $d_{G_{\infty} \star H_{\infty}}=d_{H_{\infty} \star G_{\infty}}$

Corollary 5. If $r_{1}=r_{2}=r_{3}=\cdots=r_{p}=r$ and $s_{1}=s_{2}=s_{3}=\cdots=s_{q}=s$ then

$$
d_{G_{\infty} \star H_{\infty}}=-\frac{\ln (p q)}{\ln (r s)}
$$

\section{Conclusion}

In this paper, the box-counting dimension is used to compute an explicit formula for the dimension of fractals that arise from multiple contractions. This formula allows a very convenient way to compute the dimension of fractals obtained by using the convolution of two fractals. The dimension of the fractal obtained also turns to be explicit and very easy to manipulate in order to prove the properties of the dimension of the convoluted fractal. I believe that much more properties related to the dimension of a fractal can be proved easily using the box-counting dimension. Also, in this paper, two fractals are combined using the composition of functions since the composite of contractions is always a contraction. The multiplication however is not necessary a contraction. It could be interesting in the case we deal with a contraction to study the properties of the dimension of the fractal obtained by combining two fractals using the multiplication instead of the composition. The box-counting dimension might be an appropriate tool to derive any property of the dimension of the fractal obtained.

\section{Conflicts of Interest}

The author declares no conflicts of interest regarding the publication of this paper.

\section{References}

[1] Alligood, K.T., Sauer, T.D. and Yorke, J.A. (1996) Chaos: An Introduction to Dynamical Systems. Springer Verlag. https://doi.org/10.1007/b97589

[2] Ott, E. (1993) Chaos in Dynamical Systems. Cambridge University Press, Cambridge.

[3] Barnsley, M.F. and Demko, S. (1985) Iterated Function Systems and the Global Construction of Fractals. Proceeding of the Royal Society of London, Series A, Mathematical and Physical Sciences, 399, 243-275. https://doi.org/10.1098/rspa.1985.0057

[4] Falconer, K.J. (2014) Fractal Geometry: Mathematical Foundations and Applications. 3rd Edition, Wiley.

[5] Devaney, R.L. (1994) Complex Dynamical Systems: The Mathematics behind the Mandelbrot and Julia Sets. Proceedings of the Symposia in Applied Mathematics, 49.

[6] Mandelbrot, B. (2004) Fractals and Chaos: The Mandelbrot Set and Beyond. Sprin- 
ger, New York. https://doi.org/10.1007/978-1-4757-4017-2

[7] Cotton, N., McLeman, C. and Pinchock, D. (2015) On Combining and Convolving Fractals. The College Mathematics Journal, 46, 99-108.

https://doi.org/10.4169/college.math.j.46.2.99

[8] Moran, P.A.P. (1946) Additive Functions of Intervals and Hausdorff Measure. Proceedings-Cambridge Philosophical Society, 42, 15-23.

https://doi.org/10.1017/S0305004100022684

[9] Rushing, T.B. (1992) Hausdorff Dimension of Wild Fractals. Transactions of the American Mathematical Society, 334, 597-613.

https://doi.org/10.1090/S0002-9947-1992-1162104-8

[10] Wu, J., Jin, X., Mi, S. and Jang, J. (2020) An Effective Method to Compute the Box-Counting Dimension Based on the Mathematical Definition and Intervals. Results in Engineering, 6, 100106. https://doi.org/10.1016/j.rineng.2020.100106

[11] Moran, M. and Rey, J.-M. (1998) Singularity of Self-Similar Measures with Respect to Hausdorff Measures. Transactions of the American Mathematical Society, 350, 2297-2310. https://doi.org/10.1090/S0002-9947-98-02218-1 